\title{
Dyad and Triad Census Analysis of Crisis Communication Network
}

\author{
Shahadat Uddin ${ }^{*}$, Liaquat Hossain \\ The University of Sydney, Sydney, Australia \\ Email: *shahadat.uddin@sydney.edu.au
}

Received November 11, 2012; revised December 12, 2012; accepted January 11, 2012

\begin{abstract}
Dyad and triad census summarize much of the network-level structural information of a given directed network. They have been found very useful in analyzing structural properties of social networks. This study aims to explore crisis communication network by following dyad and triad census analysis approach to investigate the association of micro-level communication patterns with organizational crisis. This study further tests hypothesis related to the process of data generation and tendency of the structural pattern of transitivity using dyad and triad census output. The changing communication network at Enron Corporation during the period of its crisis is analyzed in this study. Significant differences in the presence of different isomorphism classes or micro-level patterns of both dyad and triad census are noticed in crisis and non-crisis period network of Enron email corpus. It is also noticed that crisis communication network shows more transitivity compared to the non-crisis communication network.
\end{abstract}

Keywords: Dyad Census; Triad Census; Crisis Communication Network; Organizational Crisis

\section{Introduction}

Communication networks are represented as patterns of contacts which are created due to the flow of messages among participating actors or communicators through time and space. The word "message" encompasses everything (e.g. data, information, knowledge, image and symbol) that can be co-created by network members and that can flow from one point of contact to another within and between networks. In contemporary organizations, a communication network could take various forms such as personal contact network, work related contact network, strategic alliances among various firms, global network of organizations, and flow of information within and between groups [1].

By applying dyad and triad census analysis approach this study explores the changing micro-level structure in order to investigate patterns associated with the final stage of a crisis communication network. The dyad census seeks the tendency of reciprocity of relations within a communication network. Frequencies of different microlevel patterns are examined in triad census analysis. Both dyad and triad census analysis have gained noticeable popularity in recent social network research $[2,3]$.

Crisis communication network of an organization is the context of this study. A crisis communication network can be defined as the network that has been evolved among actors (e.g. staff) during an organizational ${ }^{*}$ Corresponding author. crisis period. Organizational crisis has been defined in many ways by many different researchers such as organizational mortality, organizational death, organizational exit, bankruptcy, decline, retrenchment and failure to characterize various forms of organizational needs [4]. Although there is limited consensus among researchers on the precise definition of organizational crisis, there is evidence of shared meaning. Hermann [5] defined crisis as a situation that threatens goals of an organization, surprises the decision makers by its occurrences, put them under time pressure for appropriate responses and consequently engender high level of stress. Milburn et al. [6] identified several important elements of an organizational crisis such as: organizational crisis produces individual crisis; crisis can be associated with positive or negative condition; crises can be situations having been precipitated quickly or suddenly or situations that have developed over time; and crises are predictable. Weitzel and Johnson [7] defined organizational crisis as a state in which firms fail to anticipate, recognize, avoid, neutralize, or adapt to external or internal pressures that threaten the organization's long term survival. Sheppard [8] defined crisis as "a critical and irreversible loss by the system" and posited that an organization dies when it stops performing its expected functions. A drastic form of critical loss occurs when firms moves into bankruptcy as in the case of Enron Corporation in the final quarter of 2001.

This study starts with the premise that email networks 
constitute a useful proxy for the underlying communication networks within organizations. A study by Smith et al. [9] investigated how different age groups managed their personal networks and what types of technologymediated communication tools they used. They found that people around their 30s (25 - 35 years) used email with the most of their social network contacts $(81 \%)$. $60 \%$ of older age groups (50 - 60 years) also tended to keep in touch with their personal contacts primarily by using emails. Wellman [10] argued that computer supported social networks (CSSNs) sustain strong, intermediate and weak ties that provide information and social support in both specialized and broadly-based relationships. CSSNs support and foster both formal and informal workplace communities. Guimera et al. [11] argued that the email network provides an inexpensive but powerful alternative to traditional approach of survey which is expensive and time consuming. Indeed, they found that the exchange of email between individuals in organizations reveals how people interact and facilitates mapping informal networks in a non-intrusive, objective and quantitative way. Tyler et al. [12] described email communication network as a tantalizing medium for research which offers a promising resource for tapping into dynamics of information within organizations and for extracting hidden patterns of collaboration and leadership that are at the heart of informal communities of practice. As a modern and technologically advanced organization, it is well known that employees of Enron (i.e. the research subject of this study) used email as a significant medium of communication.

This paper is organized as follows: in the next two sections dyad and triad census are discussed as a way to analyze communication networks. An overview of Enron email corpus that is analyzed in this study is described in the subsequent section. This is followed by a discussion of results and their implications. Finally there is a conclusion of this research.

\section{Dyad and Dyad Census}

A dyad consists of an unordered pair of actors and links that exist between two actors of the pair. Dyads are 2-subgraphs where a subgraph is a subset of actors taken from the complete set of network actors and all links between them [13]. The dyad consisting of actors $i$ and $j$ is denoted by $D_{i j}=\left(X_{i j}, X_{j i}\right)$, for $i \neq j$ and where $X$ represents the sociomatrix of the complete directed network under consideration. For a network of size $g$, there are exactly

$$
\left(\begin{array}{l}
g \\
2
\end{array}\right)=g *(g-1) / 2 \text { dyads. }
$$

The dyadic relation between any two actors in a given network must have one of the three possible states or isomorphism classes as illustrated in the Figure 1. By

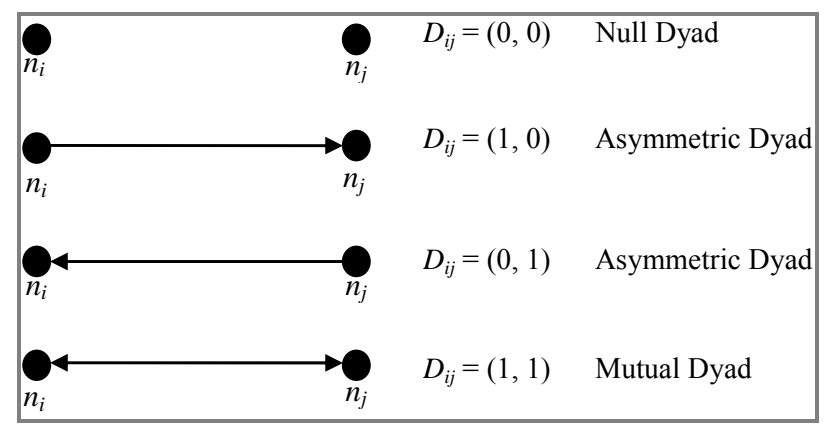

Figure 1. Three dyadic isomorphism classes or states.

definition, two subgraphs are isomorphic if they are identical. That means there exists a one-to-one mapping among actors for these two subgraphs, except the possibility of different labeling of those actors [14]. As presented in the Figure 1, a mutual relationship, denoted by $i \leftrightarrow j$, between actor $i$ and actor $j$ exists when $i \rightarrow j$ and $j$ $\rightarrow i$ in the dyad. In the sociomatrix $X$, the two symmetrical cells $(i, j)$ and $(j, i)$ are unity (i.e. $X_{i j}=1$ and $X_{j i}=1$ ) for this type of dyadic relation. That is why, the mutual dyadic relation between actor $i$ and actor $j$ is represented by $D_{i j}=(1,1)$. The second state is the asymmetric dyad which can occur in two ways: either $i \rightarrow j$ or $j \rightarrow i$, but not in both ways. The asymmetric dyadic relation between actor $i$ and actor $j$ is represented by $D_{i j}=(1,0)$ or $(0,1)$. In the sociomatrix $X$, one of the two symmetrical cells (i.e. $X_{i j}$ and $X_{j i}$ ) contains a 1 for this kind of dyadic relation. Since the labeling in the sociomatrix is arbitrary, it is not possible to distinguish two different forms (i.e. $i$ $\rightarrow j$ and $j \rightarrow i$ ) of asymmetric dyadic relations. The third state is the null dyad, in which neither actor has a tie to the other. By default, a dyad that is not asymmetric or mutual must be null. For null dyad, two symmetrical cells (i.e. $X_{i j}$ and $X_{j i}$ ) in the sociomatrix $X$ contain a 0 . That means, for null dyad $X_{i j}=X_{j i}=0$, and $D_{i j}=(0,0)$.

If $M, A$ and $N$ are numbers of mutual, asymmetric and null dyads in a collection of $\left(\begin{array}{l}\mathrm{g} \\ 2\end{array}\right)$ dyads then these three counts sum to $\left(\begin{array}{l}\mathrm{g} \\ 2\end{array}\right)$ because they provide a complete partition of the collection of dyads for any given directed network of size $g$. The triple $<M, A, N>$ is called the dyad census. The frequencies of $M, A$ and $N$ can be calculated directly from the element of the sociomatrix $X$ for any given directed network under study by the following equations:

$$
\begin{gathered}
M=\sum_{\mathrm{i}<\mathrm{j}} X_{i j} X_{j i} \\
A=X_{++}-2 M \\
\mathrm{~N}=\left(\begin{array}{l}
\mathrm{g} \\
2
\end{array}\right)-A-M
\end{gathered}
$$

where, $X_{++}=L$, number of links in the network. 


\subsection{Katz and Powell Index for Mutuality}

In order to measure the tendency for actors in a group to reciprocate choices more frequently than it would occur simply by chance, Katz and Powell [15] proposed an index which was named according to their names-Katz and Powell Index for Mutuality $\left(\rho_{\mathrm{kp}}\right)$. Like other statistical indices, this index is dimensionless and has the range of $-\infty<\rho_{\mathrm{kp}} \leq 1$; where 0 indicates no tendency for reciprocation, 1 represents maximal tendency for reciprocation, negative values indicate tendencies toward asymmetric and null dyads.

Based on the assumption that choices are made by actors in some random manner, Kat and Powell [15] normalize this index for two particular network data collection designs: fixed choice and free choice. While no restriction is placed on the number of actors each actor can relate to in a free choice design, the investigator gathers data or instructs each respondent to name a fixed number of others that the actor relates to on the relation under study in a fixed choice design. For a fixed choice design, if $d$ is the fixed number of choices made by each of the $g$ actors then $\rho_{\mathrm{kp}}$ can be estimated by the following equation [15]:

$$
\hat{\rho}_{k p}=\frac{2(g-1) M-g d^{2}}{g d(g-1-d)}
$$

In free choice designs, choices made by different actors are not necessarily equal. Katz and Powell [15] derived the following equation to estimate $\rho_{\mathrm{kp}}$ in a free choice design:

$$
\hat{\rho}_{k p}=\frac{2(g-1)^{2} M-L^{2}+L_{2}}{L(g-1)^{2}-L^{2}+L_{2}}
$$

where, $\mathrm{L}=\sum \mathrm{x}_{\mathrm{i}+}$ is the total number of choices, and $\mathrm{L}_{2}=\sum \mathrm{x}_{\mathrm{i}+}^{2}$ is the sum of squares of the choices. And, $x_{i+}$ represents the number of choices made by the $i^{\text {th }}$ actor.

\section{Triad and Triad Census}

A triad or 3-subgraph is a set of three actors $n_{i}, n_{j}$ and $n_{k}$ where $i \neq j \neq k$ [14]. While a triad could be without any tie between its three constituent actors, there could be links between the three actors of a triad. There are exactly

$$
\left(\begin{array}{l}
\mathrm{g} \\
3
\end{array}\right)=\mathrm{g} *(\mathrm{~g}-1) *(\mathrm{~g}-2) / 6
$$

triads for a network of size $g$.

As three actors constitute a triad and each actor can relate to other two actors, there are six possible ties or links between actors. In the mathematical presentation of a triad, each of these six arcs can be present or absent.
Thus, there are $2^{6}=64$ realizations or possible states for a triad if node labels are considered. However, some of these 64 states are isomorphic or structurally indistinguishable if node labels are ignored. There are sixteen isomorphism classes for the 64 different triad states [16], which are pictured in Figure 2. The triad census consists of these sixteen isomorphism categories. Like dyad census, models based on triad census can be used to test the presence of configurable biases such as transitivity bias.

\section{Research Dataset}

This study considers Enron email communication data of the year 2001 as the data for crisis communication network. In order to fully understand the context of this research, it is required to understand Enron's organizational downfall, mostly instigated by the unethical business practices of its senior management and overall organizational culture. Enron was founded in 1985 through the merger of two gas pipeline companies. Within a decade, this organization became a global player and a symbol of innovative and progressive business conglomerate. It also became actively involved in the area of metals, pulps and paper, broadband assets, water plants and financial markets internationally [17]. In the year 2000, Enron's annual revenue was $\$ 101$ billion which made it the seventh largest company in the United States, bigger than IBM or Sony [18]. On mysterious circumstances, Jeff Skilling resigned as CEO on August 14,

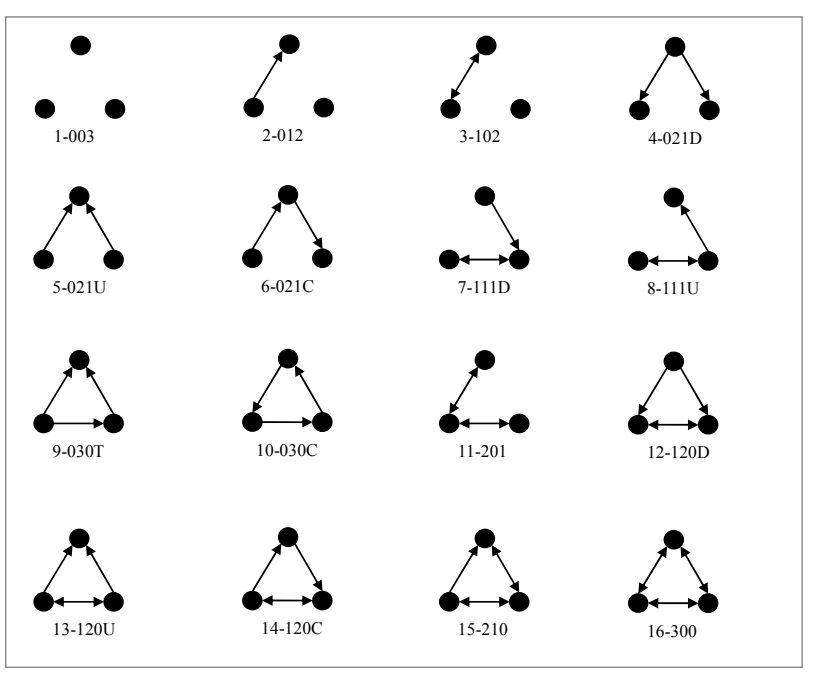

Figure 2. Sixteen triad isomorphism classes. The number (i.e. 1 to 16) before the hyphen for each labeling represents the triad ID number. The characters after the hyphen follow standard M-A-N labeling convention: the first character gives the number of mutual dyads, the second character gives the number of asymmetric dyads, the third character gives the number of null dyads and the last character (if present) is used to distinguish further among the types of classes (" $D$ ” for down, " $U$ " for up, “T” for transitive and “ $C$ ” for cycle). 
2001 and was replaced by Kenneth Lay, the founder. During the same month, it became slowly evident that, with the help of Arthur Andersen (Enron's auditor since 1985), Enron had been grossly overstating its profits and understating debts for the previous 5 years. On October 16,2001 , Enron disclosed that it had lost $\$ 618$ million in the third quarter earnings. On December 2, 2001, Enron filed for chapter 11 bankruptcy protection in a New York Bankruptcy court. With $\$ 62$ billion in assets, this was the largest bankruptcy in the history of the US up to that time. By January 2002, Enron stock lost $99 \%$ of its value. Stockholders lost tens of billions of dollars and many of the company's 20,000 employees lost their retirement saving pensions and jobs [17-19]. Since filing for bankruptcy on December 2, 2001, the Justice Department conducted an ongoing criminal investigation into the fall of Enron. This investigation had been resulted in a number of criminal charges including fraud, conspiracy and insider trading being filed against several top executives.

In May 2002, the US Federal Energy Regulatory Commission (FERC) publicly released a large set of email messages, the Enron corpus. The corpus contains 619,446 email messages belonging to 158 users over a period of 3.5 years. Shetty and Adibi [20] of University of Southern California created a MySQL database of this corpus. They also cleaned the database by removing a large number of duplicate emails, computer generated folders, junk data, invalid email addresses and blank messages. The resulting dataset contains 252,759 messages from 151 employees distributed in and around 3000 user defined folders. In this study, this database is utilized to perform required data experiment. In the area of organizational science and social network research, the Enron corpus is of great value because it allows academics to conduct research on real-life organization over a number of years.

Even though Enron email corpus has the email communication data prior to and after the year 2001, this study considers data only for the year 2001 since Enron's organizational crisis was at its peak during this period which resulted in the bankruptcy declaration during the first week of December 2001. UCINET [21] and Pajek software were used for triad census analysis; whereas, for dyad census analysis (i.e. Katz and Powell Index) variables of Equation (5) are first measured in Microsoft Excel. After that, $\rho_{\mathrm{kp}}$ is calculated from these variables.

\section{Result}

This section presents results of dyad and triad census analysis of the crisis communication network of Enron. These results highlight the presence of different structural patterns of communication structure associated with the last stage of organizational crisis of Enron.

\subsection{Dyad Census}

The number of dyads in the communication network for each of 52 weeks of the year 2001 is first identified. The result is presented in Figure 3. Then, values for $\rho_{\mathrm{kp}}$ of the same time period (i.e. 52 weeks of the year 2001) are compared in Figure 4.

From Figures 3 and 4, it is evident that there is a sharp increase in the number of dyads and in the value of $\rho_{\mathrm{kp}}$ as the Enron's crisis communication network moves towards the peak crisis period. Although the trend is not monotonically increasing, the dramatic increase in dyads and $\rho_{\mathrm{kp}}$ during September, October and November of 2001 are significant.

\subsubsection{Testing for the Distribution of Data Generation}

Dyadic statistics are used to test hypothesis related to the process of data generation for weekly networks under study. It is assumed that weekly networks are distributed as uniform random directed graph. This assumption implies that elements of the sociomatrix $X$ for each weekly network are independent and have a constant probability of 0.5 of being unity. That is, each element of $X$ is a Bernoulli random variable. Let, $L$ is equal to the count of how many of these Bernoulli random variables are unity. Since, the sum of independent Bernoulli variables, with constant probability $P$ of being unity, is a Binomial random variable with parameters equal to number of Bernoulli random variables being summed (i.e. $\mathrm{g}(\mathrm{g}-1))$ and

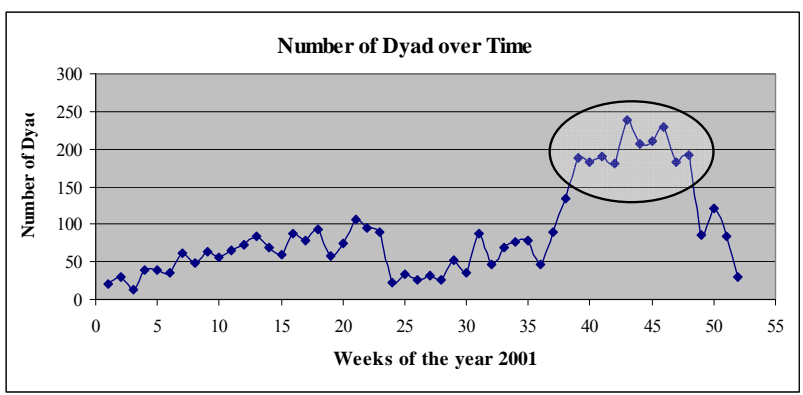

Figure 3. Change of the number of dyads in 52 different weeks of the year 2001 for the crisis communication network of Enron.

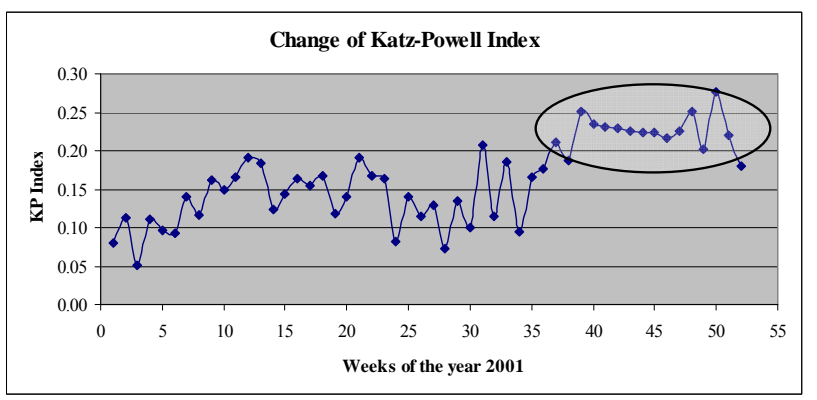

Figure 4. Change of $\rho_{\mathrm{kp}}$ in 52 different weeks of 2001 for the crisis communication network of Enron. 
the probability that any one of the variables is unity (i.e. $P)$, the null hypothesis for $L$ can be represented by the following equation [14]:

$$
\mathrm{H}_{0}: L \sim \operatorname{Bin}(\mathrm{g}(\mathrm{g}-1), 0.5)
$$

As the value for $g$ is high for the Enron email corpus, $L$ should be approximately Gaussian or normally distributed. Thus, the proposed null hypothesis can be tested by the following test statistics [14]:

$$
z_{l}=\frac{|l-E(L)|}{\sqrt{\operatorname{Var}(L)}}=\frac{|l-g(g-1) / 2|}{\sqrt{g(g-1) / 4}}
$$

where, $1=\sum_{\mathrm{i}=1}^{\mathrm{g}} \sum_{\mathrm{j}=1}^{\mathrm{g}} x_{i j}$

is the number of links actually observed in the weekly directed network under study.

Although there is a sharp increase in the $z_{l}$ values during the crisis period, as pictured in Figure 5, it is noticed that the corresponding $p$ values for all weekly networks are nearly 0 . Thus, it is highly unlikely that the data for Enron email corpus have been generated by the Bernoulli process. Therefore, it can be concluded that there is no significant difference in the data generation process during crisis and non-crisis period for Enron.

\subsection{Triad Census}

Using UCINET and Pajek, frequencies of sixteen isomorphism classes of triad census are measured for each of 52 weeks of the year 2001 of the Enron communication network. As the first three isomorphism classes of triad census (i.e. the first three structures in Figure 2) are considered in the dyad census analysis, the triad census analysis is represented in the Figure 6 by considering the rest thirteen isomorphism structures. The triad census analysis in Pajek produces both frequencies of isomorphism classes and their expected numbers. Before presenting the triad census output, it is required to normalize frequencies of triadic isomorphism classes for each weekly network by subtracting the expected number of

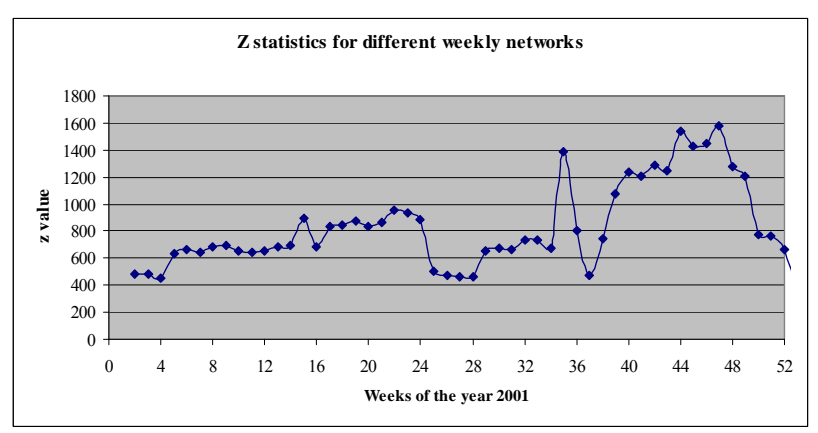

Figure 5. Zl statistics for 52 weekly networks of 2001 of the crisis communication network of Enron. the frequency from the real number of frequency for each isomorphism class. In Figure 6(a), results of those structures for which there is an absent link between actors (i.e. structures 4 to structure 8 and structure 11 in the Figure 2) are presented. The results for the rest isomorphism classes (i.e. structure 9 to structure 16 except structure 11 in Figure 2) are illustrated in Figure 6(b).

From Figures 6(a) and (b), it is revealed that there is a sharp increase in frequencies during the crisis period of Enron for all isomorphism classes. In some cases, as like for the isomorphism class of 16 - 300, evidences of high frequencies are noticed during the normal time (i.e. noncrisis period).

\subsubsection{Testing for Transitivity}

The results from the triad census analysis are further utilized to test the structural hypothesis about transitivity. Three actors (say $A, B$ and $C$ ) are transitive if whenever $A$ is linked to $B$ and $B$ is linked to $C$, then $A$ is also linked to $C$. The three actors of any transitive structure are referred as a transitive triple [22].

Out of thirteen isomorphism classes for triad census, as pictured in Figure 4, six of them have at least one transitivity configuration. They are illustrated in Figure 7. Isomorphism class $9-030 \mathrm{~T}$ and $14-210$ have one transitive triple; $12-120 \mathrm{D}$ and $13-120 \mathrm{U}$ have two transitive triples; 15 - 210 has three transitive triples; and 16 - 300 has six transitive triples. In order to count the total transitive configurations for a given directed network from its triad census, it is required to multiply the frequency of each isomorphism class by the number of transitive triple(s) being presented in its structure. For instance, if frequencies of 9 - $030 \mathrm{~T}, 12-120 \mathrm{D}, 13-120$ $U, 14-210,15-210$ and $16-300$ are 1, 2, 3, 4, 5 and 6 respectively then the total number of transitive configuration will be 66 (i.e. $1 \times 1+2 \times 2+3 \times 2+4 \times 1+5 \times$ $3+6 \times 6=66$ ). Frequencies of transitive configurations for 52 weeks of the year 2001 of Enron email corpus are compared in Figure 8.

From the Figure 8, it is evident that there is a significant increase in the number of transitive configurations in the Enron email corpus during the crisis period. Therefore, it can be concluded that crisis communication network of an organization becomes increasingly transitive as that organization experiences crisis.

\section{Discussion}

Dyad and triad census analysis have been conducted to explore 52 weekly networks of Enron email communication network. Both dyad and triad census output revealed notable changes in the pattern of communication structure during the organizational crisis period. More specifically, they showed significant increases in the occurrences of different micro-level structural patterns in 
communication network as Enron approached disintegration during the last quarter of 2001. It is important to note that this was the time during which Enron was in complete turmoil. Jeff Skilling resigned as CEO on August, 14 2001. After some time, during mid October, the company announced that it had lost $\$ 618$ million dollars in the third quarter earnings which eventually lead to the bankruptcy declaration on December 2, 2001. The bankruptcy declaration and subsequent departure of many employees lead to the reduction in the number of different micro-level dyad and triad census structures in late December.

The high frequencies of micro-level structures for dyad and triad census indicate that actors or individuals within Enron had come closer during its crisis period. The reason behind this can be explained by several existing theories. For example, Social Exchange Theory can be employed to vindicate the reciprocity within communication network. This theory, which was originally introduced by Homans [23,24] seeks to explain the likelihood of a reciprocal or dyadic relationship based on the
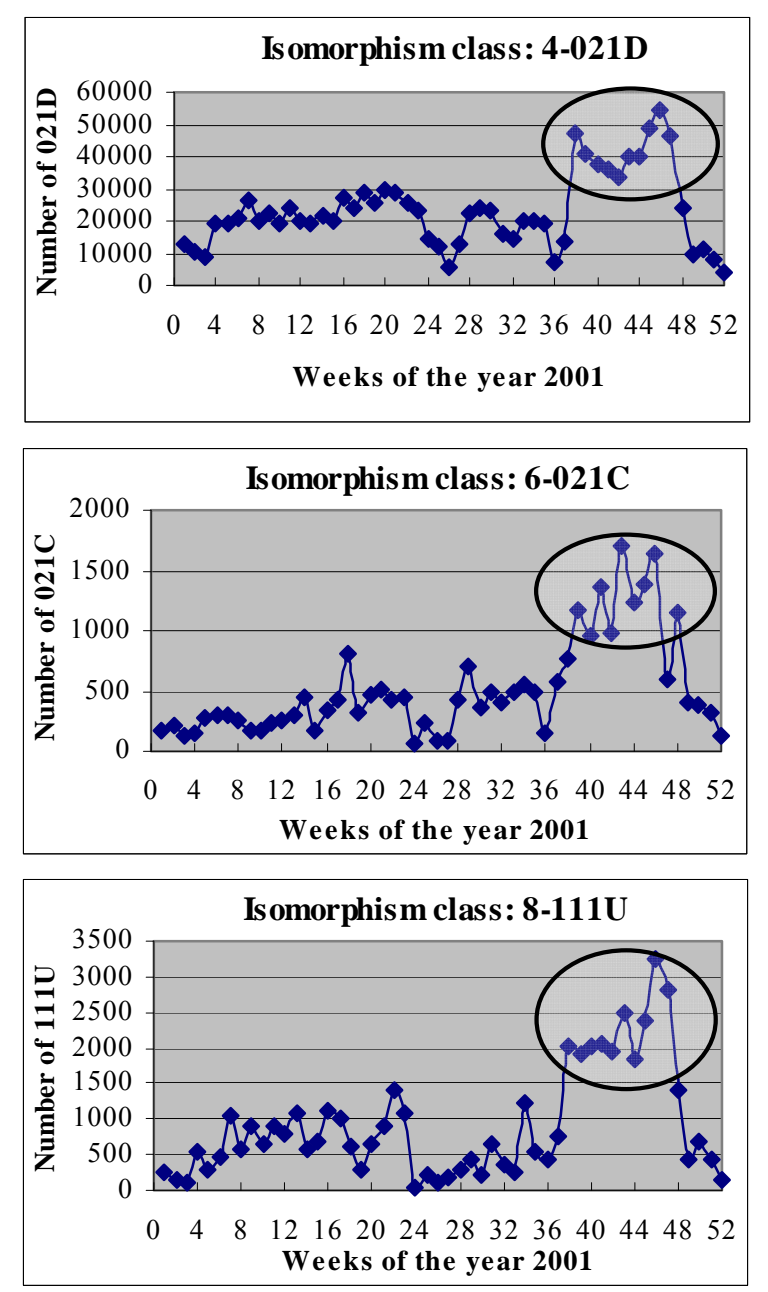

exchanges of resources, both psychological and economical, between each member of the dyad. Social Support Theory, which was proposed by Kadushin and Kadushin [25] and Lin and Ensel [26], can explain theoretical mechanisms behind actors' getting closer in the communication network during crisis. According to this theory, intimate relations with others whom one might confide and receive various forms of feedback may significantly affect one's well-being. Murshed et al. [27] described how the formation of transitive structure, which has a striking resemblance to the concept of Balance Theory, in the communication network during crisis can be explained by the Balanced Theory. They argued that people prefer balanced structure in their day to day lives. If the structure is not balanced then people experience various psychological effects such as "strain" and "tension". As organizations go through the state of crisis, people also experience "strain" and "stresses", which will ultimately lead actors to form a balanced state within the communication structure.

There are also many well-known and highly cited
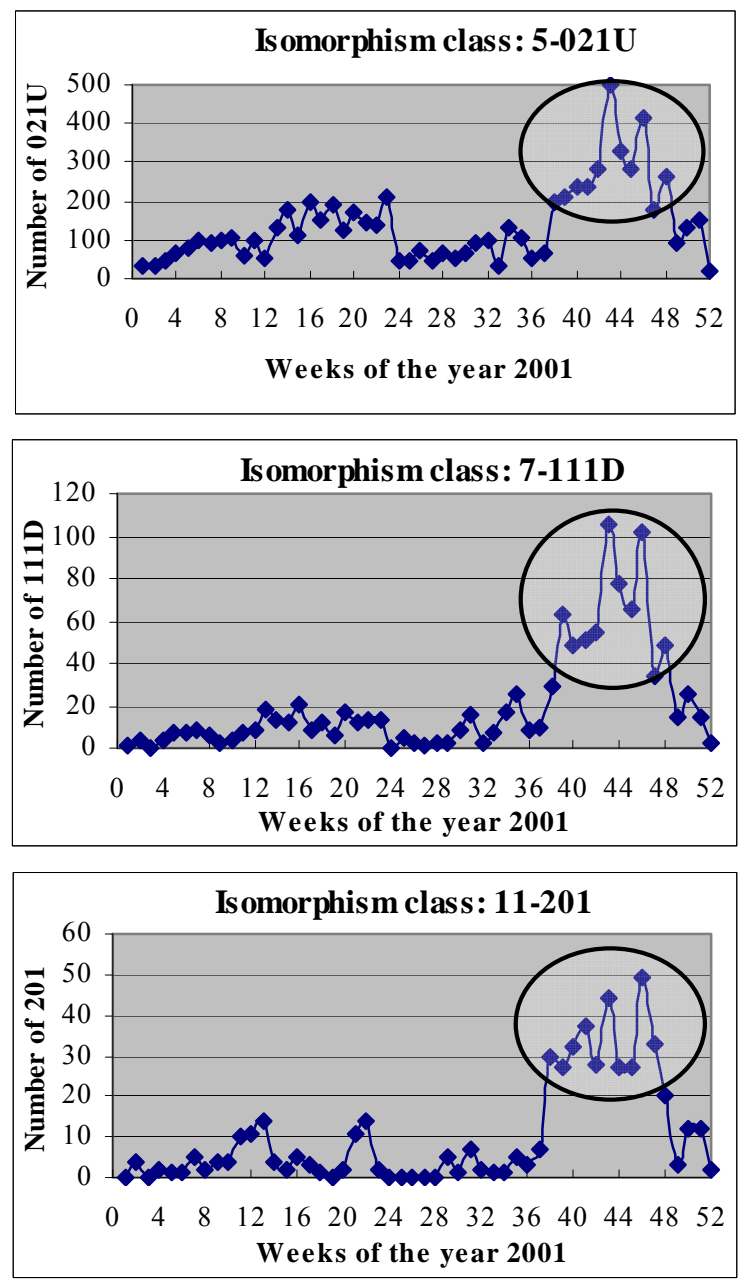

(a) 

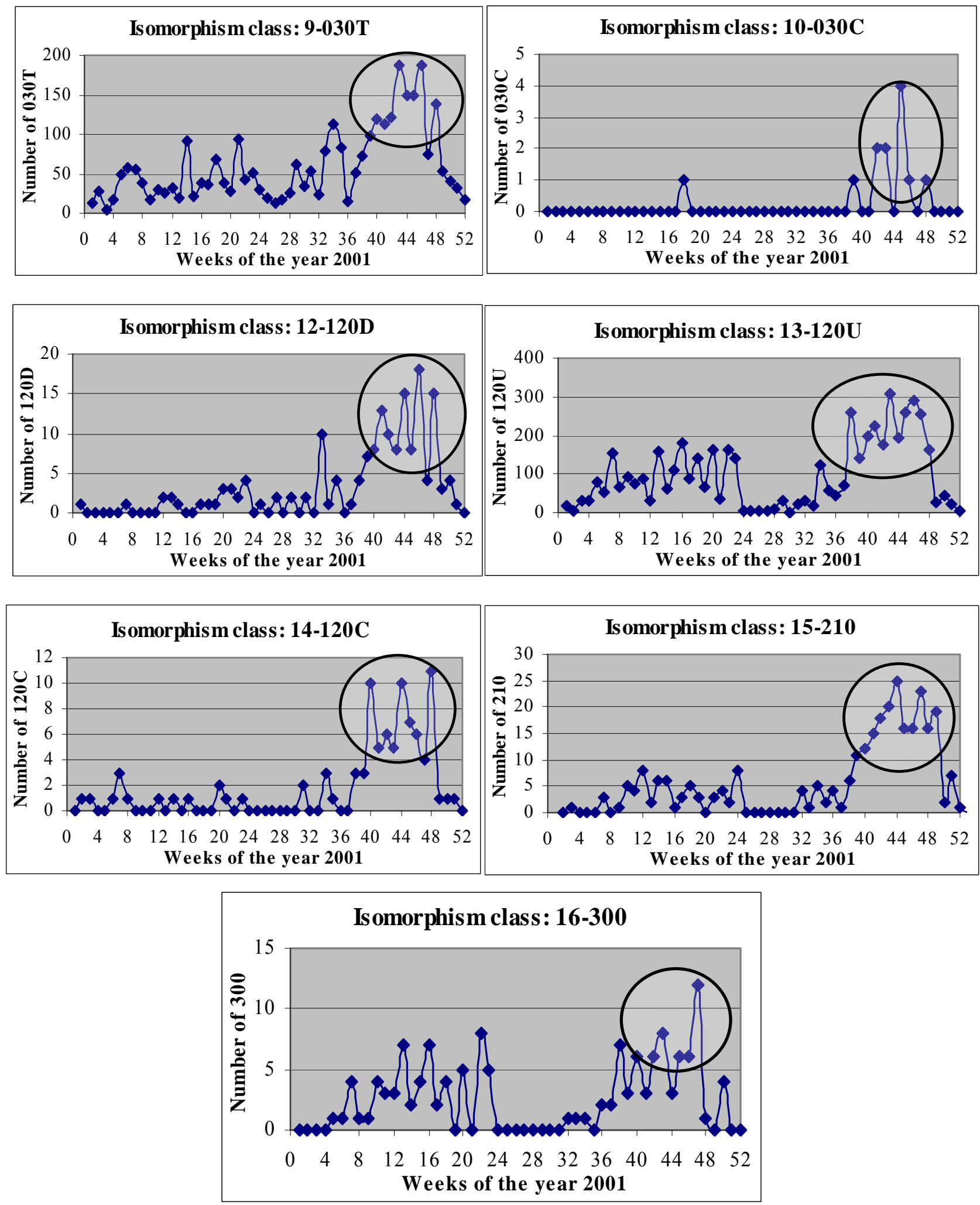

(b)

Figure 6. (a) Changes of the number of different isomorphism structures for which there is an absent link between actors (i.e. structure 4 to structure 8 and structure 11) over the 52 weeks of the year 2001 of the crisis communication network of Enron; (b). Change of the number of different isomorphism structures having at lest one link between actors (i.e. structure 9 to structure 16 except structure 11) over the 52 weeks of the year 2001 of crisis communication network of Enron. 


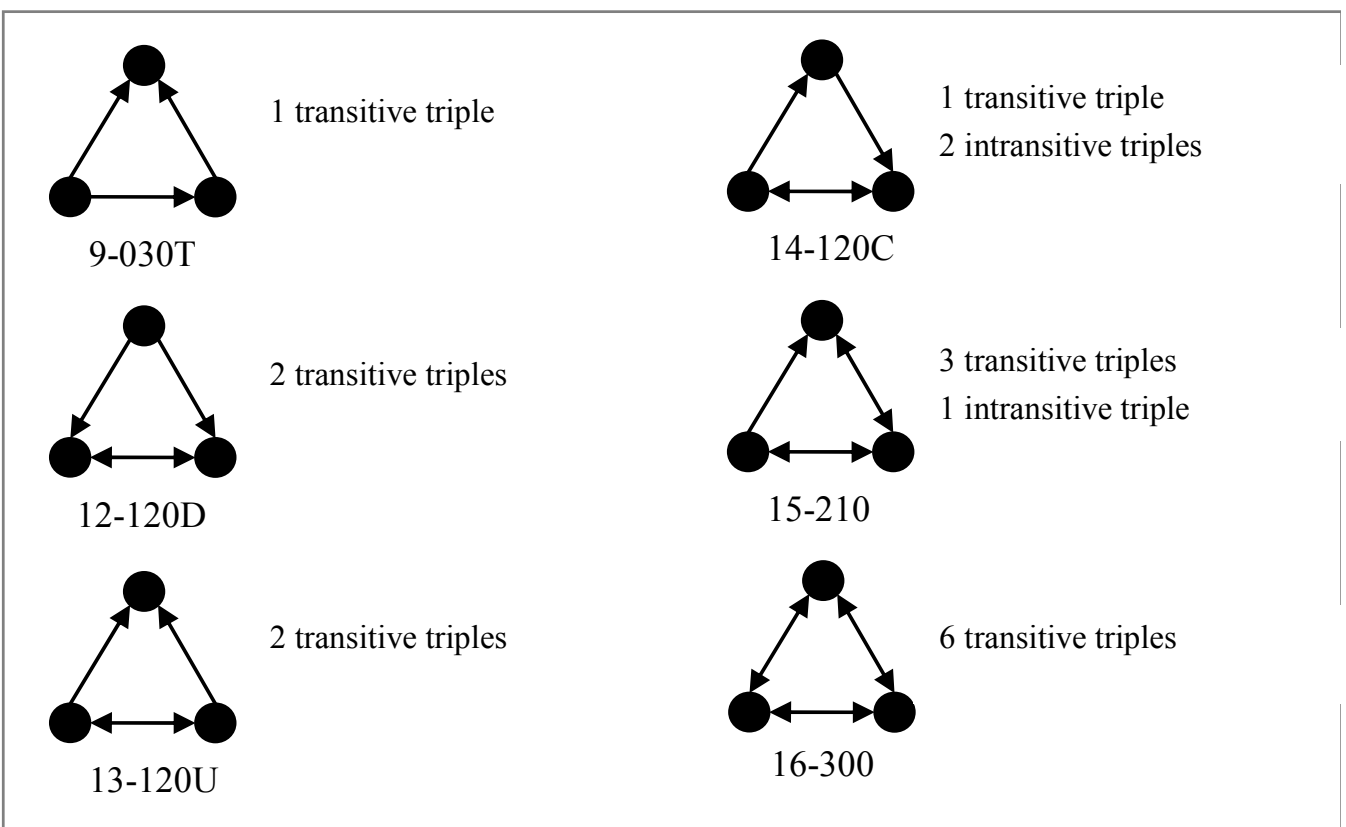

Figure 7. Statistics about transitive and intransitive triples for each of the six transitive configurations that are members of sixteen triadic isomorphism classes.

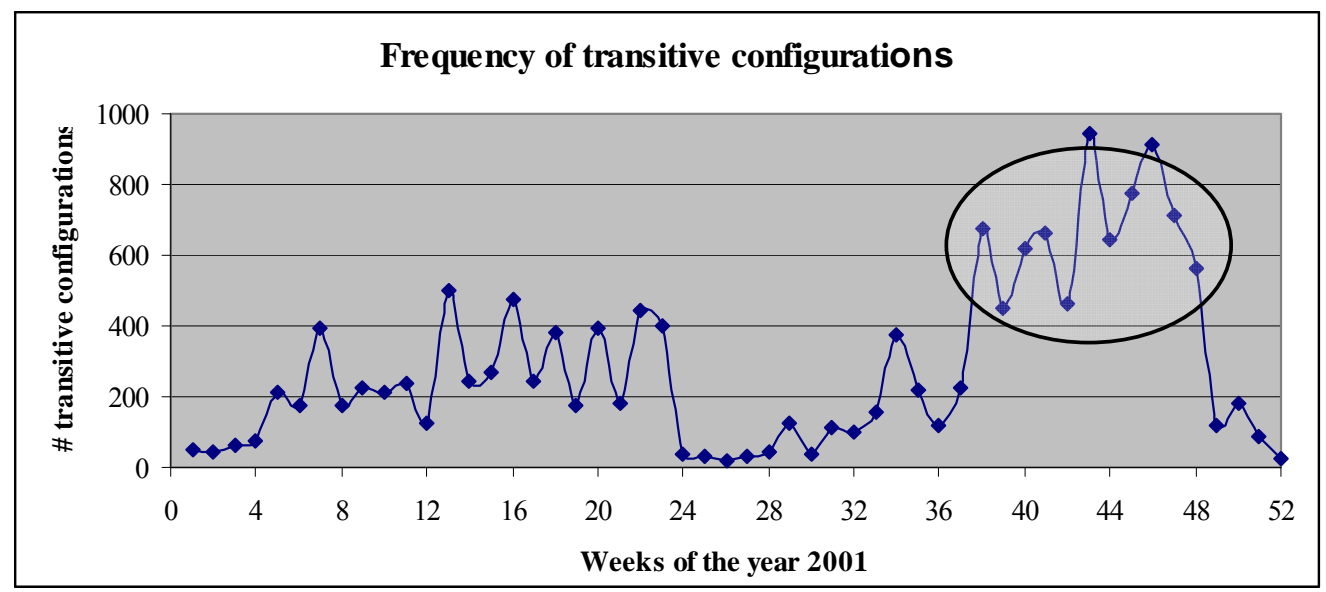

Figure 8. Frequencies of transitive configurations for 52 weeks of the year 2001 for crisis communication network of Enron.

studies in the current literature which argued that during crisis: there is a decrease in interpersonal friction and an increase in collaboration [28]; an increased cohesion among actors and a high possibility to bring people together who would otherwise have nothing to do each other [29]; an increased tendency of seeking company of others [30]; and a reduction in inter-group conflicts [31]. All of these impacts of crisis bring actors or individuals within organizations closer, which eventually make the communication network among them denser as noticed from the dyad and triad census analysis for Enron email communication data in this study.

\section{Conclusion}

This paper compares frequencies of different micro-level structures of dyad and triad census for Enron email communication network of the year 2001. Associations of different patterns with the Enron crisis period, which mainly started at the beginning of last quarter of 2001, have been noticed in this study. In dyad census analysis, higher value for Katz and Powell Index for Mutuality is observed during the organizational crisis period. Higher frequencies of different isomorphism classes of triad census are noticed during the Enron crisis period. Hypotheses related to data generation process and tendency to certain structural patterns are further tested using results from dyad and triad census analysis respectively.

The methodological contribution of this study is worthy of note. This study utilizes dyad and triad census analysis to explore crisis communication network of or- 
ganizations. With the increasing popularity of email as an interaction medium and increased popularity of social network analysis methods and tools, it is expected that a deeper understanding of the various social and organizational phenomena using further concepts of dyad and triad census analysis such as subgraph analysis, distribution analysis and stability analysis could be developed. Unlike studies that explored structural behaviors such as power-law behavior [32] and longitudinal topology of network dynamics [33,34], this study explores the presence of different micro-structures of dyad and triad census analysis for a crisis communication network.

\section{REFERENCES}

[1] P. Monge and N. Contractor, "Theories of Communication Networks," Oxford University Press, New York, 2003.

[2] M. Rustin, "Structural and Unconscious Implications of the Dyad and Triad: An Essay in Theoretical Integration; Durkheim, Simmel, Freud," The Sociological Review, Vol. 19, No. 2, 2011, pp. 179-201. doi:10.1111/j.1467-954X.1971.tb00626.x

[3] S. Fukuzumi, K. Ohkubo, J. Ortiz, A. M. Gutierrez, F. Fernandez-Lazaro and A. Sastre-Santos, "Control of Photoinduced Electron Transfer in Zinc PhthalocyaninePerylenediimide Dyad and Triad by the Magnesium Ion," The Journal of Physical Chemistry A, Vol. 112, No. 43, 2008, pp. 10744-10752. doi:10.1021/jp805464e

[4] K. Mellahi and A. Wilkinson, "Organizational Failure: A Critique of Recent Research and a Proposed Integrative Framework," International Journal of Management Reviews, Vol. 5, No. 1, 2004, pp. 21-41. doi:10.1111/j.1460-8545.2004.00095.x

[5] C. Hermann, "Some Consequences of Crisis Which Limit the Viability of Organizations," Administrative Science Quarterly, Vol. 8, No. 1, 1963, pp. 61-82. doi: $10.2307 / 2390887$

[6] T. Milburn, R. Schuler and K. Watman, "Organizational Crisis," Part I: Definition and Conceptualization. Human Relations, Vol. 36, No. 12, 1983, pp. 1141-1160. doi:10.1177/001872678303601205

[7] W. Weitzel and E. Jonsson, "Decline in Organizations: A Literature Integration and Extension," Administrative Science Quarterly, Vol. 34, No. 1, 1989, pp. 91-109. doi: $10.2307 / 2392987$

[8] J. Sheppard, "Strategy and Bankruptcy: An Exploration into Organizational Death," Journal of Management, Vol. 20, No. 4, 1994, pp. 795-833.

[9] H. Smith, Y. Rogers and M. Brady, "Managing One's Social Network: Does Age Make a Difference," Human-Computer Interaction-INTERACT, Zurich, 2003, pp. 551-558.

[10] B. Wellman, "For a Social Network Analysis of Computer Networks: A Sociological Perspective on Collaborative Work and Virtual Community," Association for Computing Machinery, 1996.
[11] R. Guimera, L. Danon, A. Diaz-Guilera, F. Giralt and A. Arenas, "Self-Similar Community Structure in a Network of Human Interactions," Physical Review E, Vol. 68, No. 6, 2003, pp. 1-4. doi:10.1103/PhysRevE.68.065103

[12] J. Tyler, D. Wilkinson and B. Huberman, "E-mail as Spectroscopy: Automated Discovery of Community Structure within Organizations," The Information Society, Vol. 21, No. 2, 2005, pp. 143-153. doi:10.1080/01972240590925348

[13] P. Carrington, J. Scott and S. Wasserman, "Models and Methods in Social Network Analysis," Cambridge University Press, Cambridge, 2005. doi:10.1017/CBO9780511811395

[14] S. Wasserman and K. Faust, "Social Network Analysis: Methods and Applications," Cambridge University Press, Cambridge, 2005.

[15] L. Katz and J. Powell, "Measurement of the Tendency toward Reciprocation of Choice," Sociometry, Vol. 18, No. 4, 1955, pp. 403-409. doi:10.2307/2785876

[16] J. Davis and S. Leinhardt, "The Structure of Positive Interpersonal Relations in Small Groups," In M. Berger, J. Zelditch and B. Anderson, Eds., Sociological Theories in Progress, Vol. 2, 1967. pp. 218-251.

[17] P. Healy and K. Palepu, "The Fall of Enron," The Journal of Economic Perspectives, Vol. 17, No. 2, 2003, pp. 3-26. doi:10.1257/089533003765888403

[18] L. Fox, "Enron: The Rise and Fall," John Wiley \& Sons Inc., Hoboken, 2003.

[19] E. Hamilton, "An Exploration of the Relationship between Loss of Legitimacy and the Sudden Death of Organizations," Group \& Organization Management, Vol. 31, No. 3, 2006, pp. 327-358. doi: $10.1177 / 1059601106286885$

[20] J. Shetty and J. Adibi, "The Enron Email Dataset Database Schema and Brief Statistical Report," Information Sciences Institute Technical Report, University of Southern California, 2004.

[21] S. Borgatti, M. Everett and L. Freeman, "UCINET for Windows: Software for Social Network Analysis," Harvard Analytic Technologies, 2002.

[22] P. Holland and S. Leinhardt, "Holland and Leinhardt Reply: Some Evidence on the Transitivity of Positive Interpersonal Sentiment," Journal Storage, Vol. 77, No. 6, 1972, p. 1205- 1209.

[23] G. Homans, "Bringing Men Back in," American Sociological Review, Vol. 29, No. 6, 1964, pp. 809-818. doi:10.2307/2090864

[24] G. Homans, "Social Behavior as Exchange," American Journal of Sociology, Vol. 63, No. 6, 1958, pp. 597-606. doi: $10.1086 / 222355$

[25] A. Kadushin and G. Kadushin, "The Social Work Interview," Columbia University Press, New York, 1983.

[26] N. Lin and W. Ensel, "Life Stress and Health: Stressors and Resources," American Sociological Review, Vol. 54, No. 3, 1989, pp. 382-399. doi:10.2307/2095612

[27] S. H. Murshed, S. Uddin and L. Hossain, "Understanding Communication Network Cohesiveness during Organiza- 
tional Crisis: Effects of Clique and Transitivity," International Conference on Information Systems, St Louis, 2010.

[28] J. Lanzetta, "Group Behavior under Stress," Human Relations, Vol. 8, No. 1, 1955, p. 29. doi: $10.1177 / 001872675500800102$

[29] G. Simmel, "Conflict and the Web of Group Affiliations," Free Press, 1964.

[30] L. Weller, "The Effects of Anxiety on Cohesiveness and Rejection," Human Relations, Vol. 16, No. 2, 1963, pp. 189. doi: $10.1177 / 001872676301600208$

[31] M. Sherif, "In Common Predicament: Social Psychology of Intergroup Conflict and Cooperation," Houghton Mifflin Boston, 1966.
[32] S. Uddin, S. T. H. Murshed, and L. Hossain, "Power-Law Behaviour in Complex Organizational Communication Network during Crisis," Physica A: Statistical Mechanics and Its Applications, Vol. 390, No. 15, 2011, pp. 2845 2853.

[33] S. Uddin, L. Hossain, S. T. Murshed, and J. W. Crawford, "Static versus Dynamic Topology of Complex Communications Network during Organizational Crisis," Complexity, Vol. 16, No. 5, 2011, pp. 27-36. doi:10.1002/cplx.20349

[34] S. Uddin, M. Piraveenan, K. Chung, and L. Hossain, "Topological Analysis of Longitudinal Networks," Hawaii International Conference on System Sciences, Hawaii, 2013. 